\title{
Metabolomics in oncology - A fascinating travel into the mechanisms of metabolic disturbances during carcinogenesis
}

Evangelos Karamitrousis ${ }^{1 *}$, Michalis Liontos $^{2}$, Nikolaos Tsoukalas ${ }^{3}$

${ }^{1}$ Department of Medical Oncology, Aristotle University of Thessaloniki,

Papageorgiou General Hospital, Thessaloniki, Greece

'Department of Clinical Therapeutics, National and Kapodistrian University of

Athens, Alexandra Hospital, Athens, Greece

${ }^{3}$ Department of Oncology, 401 General Military Hospital, Athens, Greece

Several cellular functions have been altered during cancerous transformation, among them cellular metabolism. Intrinsic and extrinsic factors, such as environmental and genetic factors, play a key role in the metabolic pathway alterations of cancer cells and contribute to the oncogenic processes altering the cellular phenotype and molecular physiology ${ }^{[1,2]}$. Metabolomics is the large-scale study of small molecules, known as metabolites. Collectively, these small molecules and their interactions within a biological system are known as the metabolome. Metabolomics are currently used in biomarker identification for the diagnosis and prognosis of cancer and can play a crucial role in the evaluation of therapeutic interventions ${ }^{[3,4]}$.

The most prominent alteration in cancer cell metabolism is the phenomenon called "Warburg effect" or aerobic glycolysis. Cancer cells uptake glucose that in the presence of oxygen is not used through the Krebs cycle, but is instead incompletely oxidized or fermented to lactate. Unlike other oncogenic pathways that can be completely abrogated by using small molecular inhibitors or antibodies, this is not applicable to fundamental metabolic pathways. The latter would have immediate detrimental effects to the whole organism. Targeting through the level of enzymatic activity of nodal enzymes in metabolic pathways could provide therapeutic opportunities. Metabolomics can help us to identify the exact processes which a cancer cell can use a biological molecule (such as glucose) in order to produce energy or all the other essential biological molecules (such as nucleotides, lipids, and amino acids) $)^{[6,7]}$.

Metabolomics could have several applications in oncology. Metabolomics can be used for the development of sensitive biomarkers to help in early diagnosis of several tumors. For example, the metabolic profile in urine samples could be used for the early and precise diagnosis of renal carcinoma, which is a tumor that is often diagnosed at advanced stages ${ }^{[8]}$. In ovarian cancer, metabolomics can be used in order to detect the metabolic profile of serum proteins, which is different from the metabolic profile in healthy postmenopausal women ${ }^{[9]}$. Also, prostate cancer cells carry alterations in their metabolites, such as increased amounts of phosphatidylcholine and decreased amounts of branched amino acids ${ }^{[10]}$.

Metabolomics can also assist to develop new therapeutic interventions for several tumors. A decade ago it was shown that mutations in the isocitrate dehydrogenase 1 enzyme (IDH1) lead to the intracellular accumulation of the metabolite 2-hydroxyglutarate (2HG) [11]. 2HG contributes to the malignant transformation of glial cells. Currently, IDH1/2 inhibitors - namely ivosidenib and enasidenib - have been approved for patients with relapsed or refractory acute myeloid leukemia. Cervical cancer is another area that the use of metabolomics could have therapeutic implications. Through this 
technology, the metabolic profile of apoptotic cancer cells due to radiotherapy can be detected, and this can help in the comprehension of the apoptotic mechanism that is associated with radiation ${ }^{[12]}$. It is clear that all these studies can help in creating novel therapeutic approaches in cancer.

The above denote that metabolomics could enhance our diagnostic and therapeutic potentials in cancer in the near future. In the current issue of the journal, a series of articles related to the metabolism of cancer cells and consequently to metabolomics are published. More specifically, the impact of vitamin $D$ serum levels on

\section{References}

[1] Beger RD. A review of applications of metabolomics in cancer. Metabolites. 2013 Jul 5;3(3):552-574.

[2] Holmes E., Wilson ID. Nicholson JK. Metabolic phenotyping in health and disease. Cell 2008, 134, 714-717.

[3] Griffin JL., Shockcor JP. Metabolic profiles of cancer cells. Nat. Rev. Cancer 2004, 4, 551-561.

[4] Spratlin JL., Serkova NJ. Eckhardt SG. Clinical applications of metabolomics in oncology: A review. Clin. Cancer Res. 2009, 15, 431-440.

[5] Vander Heiden, M.G. Targeting cancer metabolism: A therapeutic window opens. Nat. Rev. Drug Discov. 2011, 10, 671-684.

[6] Armitage EG, Southam AD. Monitoring cancer prognosis, diagnosis and treatment efficacy using metabolomics and lipidomics. Metabolomics. 2016;12(9):146.

[7] Nagrath D, Caneba C, Karedath T, Bellance N. Metabolomics for mitochondrial and cancer studies. Biochim Biophys Acta. 2011 Jun;1807(6):650-663.

[8] Kim K, Aronov P, Zakharkin SO, Anderson D, Perroud B, Thompson IM, Weiss RH. Urine metabolomics analysis for kidney cancer detection and biomarker discovery. Mol Cell Proteomics. 2009 Mar;8(3):558-570.

[9] Odunsi K, Wollman RM, Ambrosone CB, Hutson A, McCann SE, Tammela J, Geisler JP, Miller G, Sellers T, Cliby W, Qian F, Keitz B, Intengan M, Lele S, Alderfer JL. Detection of epithelial ovarian cancer using 1H-NMR-based metabonomics. Int J Cancer. 2005 Feb 20;113(5):782-788.

[10] Teahan O, Bevan CL, Waxman J, Keun HC. Metabolic signatures of malignant progression in prostate epithelial cells. Int J Biochem Cell Biol. 2011 Jul;43(7):1002-1009. clinicopathological features and outcome in advanced pancreatic carcinoma ${ }^{[13]}$, the interactive role of breast cancer on dyslipidemia and hypertension metabolic risk according to treatment exposure and menopausal status $^{[14]}$, the prognostic value of cytokines in breast cancer, in relation to the hormonal status and obesity ${ }^{[15]}$, and the association of diabetes treatment and cancer ${ }^{[16]}$ are discussed. We believe all the articles of the journal are of great interest and will give readers the opportunity to acquaint with the novel subject of metabolomics in oncology.

[11] Dang, L., White, D., Gross, S. et al. Cancerassociated IDH1 mutations produce 2-hydroxyglutarate. Nature 462, 739-744 (2009)

[12] Lane AN, Fan TW, Higashi RM. Isotopomerbased metabolomic analysis by NMR and mass spectrometry. Methods Cell Biol. 2008;84:541-588.

[13] Mohammed AA. Impact of vitamin D serum levels on clinicopathological features and outcome in advanced pancreatic carcinoma. Forum Clin Oncol 2021; 12 (1): 20-30.

[14] Al-Zeidaneen AS, Ahmad MN , Al-Ebous AD et al. Interactive role of breast cancer on dyslipidemia and hypertension metabolic risk according to treatment exposure and menopausal status. Forum Clin Oncol 2021; 12 (1): 39-46.

[15] Talima S, Kaldas D, Kassem H et al. Prognostic value of cytokines in breast cancer: Correlation with positive hormonal status and obesity. Forum Clin Oncol 2021; 12 (1): 67-73.

[16] Azeez TA, Folorunso SA, Eguzozie EC et al. Antidiabetic drugs and the risk of cancer: beneficial, neutral or detrimental? Forum Clin Oncol 2021; 12 (1): 74-81. 